\title{
Estrategias de los médicos para hacer frente a la crisis de la profesión
}

\author{
Juan Pablo Jiménez de la J. \\ Doctors' strategies to cope with the \\ crisis in medical profession
}

This article reviews the strategies of doctors to face the crisis perceived in medical practice. The results of a descriptive study are compared with current data in medical literature. In that preliminary report, where physicians in different settings of practice were interviewed, the authors found a great variety of strategies, from passive resignation and escape behaviors, to a proactive conduct of information search and analysis of the changes under way. All groups interviewed reported the importance of systematic reflection, the need of life options, of adopting healthy life styles and spirituality, as strategies to cope with professional frustrations (Rev Méd Chile 2005; 133: 707-12).

(Key Words: Delivery of health care; Hospital-Physician relations; Physician-patient relations; Private practice)

Recibido el 4 de octubre, 2004. Aceptado el 20 de diciembre, 2004.

Trabajo financiado con fondos propios del Departamento.

Departamento de Psiquiatría y Salud Mental Oriente, Facultad de Medicina, Universidad de Chile. Santiago de Chile.

$\mathrm{L}^{\mathrm{a}}$ a crisis en la práctica de la profesión médica es un fenómeno bien documentado en los países desarrollados*. Desde luego, sus causas son complejas y ha motivado la investigación que nuestro grupo está llevando a cabo. En Gran Bretaña, éste es un asunto discutido crecientemente en los últimos veinte años, en el contexto de la reforma del sistema de salud, en especial en el ámbito de los médicos generales que trabajan en el nivel de atención primaria ${ }^{1}$. En nuestro país, el proceso de reforma de la salud ha desatado una aguda controversia entre el gremio médico y las autoridades del Estado. En esta polémica se tiende a obviar el punto de vista de la crisis en la profesión según es percibida localmente por los médicos.

En comunicaciones anteriores ${ }^{2-5}$, hemos delineado las principales dimensiones de la crisis, algunas de sus consecuencias percibidas por los médicos y el impacto en su "moral", de acuerdo con los hallazgos de nuestra investigación. El objetivo de ésta es presentar las principales estrategias que los doctores ponen en práctica para enfrentar la crisis.

La percepción de crisis en la práctica de la profesión afecta a la moral del gremio y a la sensación de bienestar subjetivo, estableciéndose

* Visítese la sección del British Medical Journal, "Doctor's Morale and Wellbeing", disponible en: http:// bmj.com/cgi/collection/doctors_morale_and_well_being.

Correspondencia a: Dr. Juan P Jiménez. Av. Salvador 486, Providencia. Fono-fax: 274 8855. E-mail: jjimenez@med.uchile.cl 
lo que podríamos llamar un estado de "malestar en la cultura" médica, donde la crisis objetiva del sistema de salud se instala en la subjetividad de los proveedores de salud más representativos, los doctores, y los obliga a desplegar estrategias personales de adaptación y supervivencia. El proceso de "afrontamiento" (coping) de una crisis ha sido bien estudiado en los pacientes que se ven sorprendidos por una enfermedad somática ${ }^{6}$.

Es bien sabido que el concepto de crisis se refiere tanto a una situación de amenaza como a una oportunidad de resolver una condición insostenible. En este sentido, nos interesó estudiar las estrategias personales de afrontamiento que los médicos despliegan frente a las dificultades percibidas, pues probablemente la maximización de algunas de ellas podrá contribuir a la resolución de la crisis por la que atraviesa la profesión. En Chile no existen estudios sobre las causas personales u organizacionales del estrés y la insatisfacción en el trabajo de los médicos, tampoco sobre las estrategias que aplican los médicos satisfechos. Revisando la literatura internacional reciente sobre el tema, se observa un cambio en el objetivo de investigación de la crisis en la profesión, desde el estudio de las causas del descontento hacia la exploración de las características de aquel grupo de médicos que se siente bien y que está satisfecho en su trabajo, a pesar de percibir la crisis de modo semejante al de sus pares insatisfechos $^{7,8}$.

Las características generales de nuestra investigación, esto es, las preguntas directrices, la muestra de médicos entrevistados, así como la metodología cualitativa aplicada para el análisis de los resultados, han sido informadas en artículos precedentes ${ }^{4,5}$. En todo caso, se trató de una investigación piloto, debiendo ser completada por un estudio con una muestra mayor y más representativa. En lo que sigue se revisarán las principales estrategias que los médicos entrevistados han aplicado en el afrontamiento de la crisis percibida, ordenándolas de acuerdo con las dimensiones y categorías que consigna la literatura especializada.

1. La evitación o no afrontamiento como estrategia. El mayor grado de resignación y pasividad frente a los cambios en la profesión lo encontra- mos en el grupo de médicos mayores de 50 años. Expresiones como: “¿Estrategia?, ninguna. Ser arrastrados por la ola economicista, sin remedio y sin posibilidad ninguna de cambio" o, [sólo nos queda] "la estrategia del 'sálvese quien pueda' sin priorizar el cómo...", hablan por sí solas. La percepción de que el cambio no se puede detener ni dirigir, y donde lo único que cabe es emprender acciones de escape, está expresada en la siguiente frase: "Uno no puede detenerse ante el cambio. No enfrentamos toda la frustración, la sublimamos, por ejemplo, jugando tenis". Del mismo modo, existe la percepción que los médicos deben retraerse a pequeños territorios donde puedan mantener su iniciativa, aceptando que los cambios son impuestos desde fuera: "La reforma que se avecina se va a hacer en el Ministerio de Economía y no en el de Salud. Tal vez hay que conformarse con una parcela pequeña en la cual uno haga lo que uno puede hacer".

2. Los médicos necesitan intimidad. Estudios realizados en otros países ${ }^{9}$ muestran que los médicos que mantienen relaciones íntimas satisfactorias son los que mejor toleran el estrés laboral. Al contrario, suele suceder que responsabilidades profesionales o trastornos de personalidad dificulten el logro de este objetivo, ya que es del todo claro que las relaciones requieren de cuidado y de paciencia. En este sentido, se insiste en que la profesión médica precisa un cambio cultural en contra de la creencia de que la vocación médica debe prevalecer por sobre la vida familiar. En nuestra muestra, quienes estaban más conscientes de esto fueron los doctores cuya edad promedio fue de 48 años, es decir, aquellos con hijos adolescentes. Uno de ellos manifestó: [frente a las dificultades] "refuerzo los vínculos con mis hijas..." Otro reconoció, no sin ambivalencia, apoyarse en su mujer: "La pareja es fuente de satisfacción... pero también de conflicto". Para un médico de 60 años, "se hace importante el tiempo dedicado deliberadamente a la familia".

3. Autocuidado y actitud positiva. Cualquier enfrentamiento positivo a una crisis empieza por reconocer las dificultades. Médicos y estudiantes de medicina muestran grados de perfeccionismo mayores que la población general ${ }^{10}$. En este 
sentido, la responsabilidad personal por el bienestar incluye el desafío por el cambio del pensamiento perfeccionista ${ }^{11}$, la práctica de la autorreflexión -que significa poner atención intencional a los pensamientos, sentimientos y juicios propios mientras se está con los demás-12, el cultivo de la toma de conciencia y la expresión de las emociones ${ }^{13}$, la clarificación de nuestros valores y opciones ${ }^{14} \mathrm{y}$ el aprender a hacer frente a estresores extraordinarios como, por ejemplo, fracasos terapéuticos importantes o juicios por mala práctica ${ }^{15}$. En nuestra muestra, algunos médicos, especialmente aquellos en la edad media de la vida, reconocen la necesidad de ayuda especializada: "voy al psiquiatra, tengo terapia. Disminuye la ansiedad que produce toda esa incertidumbre y eso de aperrar" o "uno tiene que buscar ayuda en otra parte... psicólogos, psiquiatras..." Los médicos más jóvenes, en especial los que trabajan en atención primaria, manifestaron una actitud francamente positiva frente a los cambios: "Para mí los cambios han sido positivos, han sido más oportunidades que amenazas". También buscan adaptarse de manera activa: "Capacitarse en el uso de nuevas herramientas, por ejemplo, en 'Medicina basada en la evidencia', para mejorar la propia posición frente a un ambiente cambiante y dinámico", o intentan entender mejor el sentido de los cambios: "estudiar y aprender más sobre 'los sistemas de salud' y las fuerzas detrás de ellos, me ha ayudado a enfocar mejor mis energías". Claro que también hay médicos mayores que se muestran entusiasmados: "Creo que hay que tener una actitud de vida positiva. La nueva tecnología es fantástica. No hay que ser pesimista"; u otros que, después de una crisis, optan por reintegrarse: "Decidí no jubilarme, estoy dispuesto a aceptar estrategias y a participar".

4. Reflexión personal sistemática. Se sabe que el hábito de reflexión personal promueve el bienestar del médico. En Gran Bretaña, grupos de discusión (tales como Doctoring to Heal ${ }^{13}$ ) sobre tópicos existenciales y espirituales en medicina son muy concurridos y apreciados, tanto por académicos como por médicos residentes, pues el compartir historias personales y profesionales suele conducir a un sentido de identidad más fuerte y a una mejor comunicación entre colegas. Durante la realización de uno de nuestros grupos focales, un médico universitario mayor de 60 años, se refirió a la importancia de reflexionar sistemáticamente: "Debiera promoverse este tipo de discusión para participar, tener presencia y para que se escuche nuestra voz". Tanto médicos jóvenes como mayores destacaron la necesidad de "revisar los fundamentos del quehacer y de reflexionar con colegas empáticos respecto del tema", y de "combinar acción y reflexión". Por su parte, un médico con trayectoria universitaria opinó que la formación académica "ayuda a mantener una reflexión sistemática sobre el sistema médico".

5. Espiritualidad, opciones de vida y bienestar. El bienestar personal y profesional también se relaciona con la espiritualidad ${ }^{16}$, entendida ésta como una cualidad que va más allá de la afiliación religiosa, y que apunta hacia la inspiración, la reverencia, el temor, el sentido y propósito, aun en aquellos que no creen en Dios. La dimensión espiritual trata de estar en armonía con el universo, se esfuerza por encontrar respuestas y, esencialmente, se transforma en foco de atención en períodos de estrés emocional, enfermedad física y mental, pérdidas, duelos y muertes ${ }^{17}$. La dimensión espiritual, para los pacientes, sus familias, y también para los médicos tratantes, es especialmente relevante cuando los doctores se encuentran frente a situaciones clínicas intratables o que conducen inevitablemente al fracaso de los tratamientos y finalmente a la muerte. Muchos médicos valoran el apoyo y el rito de cierre que se logra por un servicio religioso formal en memoria de los pacientes que mueren. Existe un creciente cuerpo de evidencias que iluminan la relación entre las variables espirituales y el bienestar emocional ${ }^{18}$. Nuestro estudio muestra que nuestros médicos, en especial los mayores, no son ajenos a reconocer la dimensión espiritual de la profesión: "Tener claro el sentido de la vida: La pregunta por la trascendencia no es una preocupación retórica y los cambios experimentados por los profesionales médicos muestran que el modo de vivir es mirando hacia adelante y arriba, más que hacia adentro y abajo". Desde luego, una actitud espiritual se acompaña de una actitud más modesta y menos omnipotente: "He adoptado la conducta 
de reconocer mi ignorancia y limitaciones, tratando de ayudar y consolar a pesar de ellas". Médicos que han superado bien la crisis de la edad media de la vida -y con ello han trasladado el locus de control hacia el interior de ellos mismos-, reconocieron haber tomado opciones fundamentales en relación con las expectativas de éxito profesional y de mayores ingresos: "No le echemos la culpa a otros de nuestros problemas personales. Yo nací "en pelotas" y moriré "en pelotas", para qué querer más. El que busca el dinero no obtiene la felicidad. Hay que hacer opciones". En el mismo sentido, uno planteó que "hay mecanismos compensadores pese a todo este nivel de estrés, bajos recursos, andar corriendo... yo diría que soy un gallo feliz... Lo primero es la paz con uno mismo".

6. La vuelta a lo esencial como estrategia. En estrecha relación con lo anterior, una estrategia no infrecuente, en especial en los médicos mayores, es intentar volver a lo que ellos consideran lo esencial de la medicina y de la vocación de servicio. Un estado de reflexión y silencio interior centrado en el estudio, junto a una actitud ética: "Desarrollar una 'cultura de monasterio', es decir, dedicarse con pasión a hacer bien la práctica médica o investigación, y así conectarse con el mundo de la ciencia en el campo de la medicina sin fronteras". Renovar el interés por la clínica y por los pacientes: "Acentuar el uso de la clínica clásica... Usar el mínimo de exámenes y procedimientos... Aumentar el tiempo dedicado a los pacientes para escuchar sus problemas y sugerir soluciones aun cuando no sean médicas". No faltó la alusión nostálgica a lugares y tiempos pasados donde se supone imperaban los valores clásicos: "Pienso en jubilarme y volver al policlínico rural donde está esa Señora. Esa relación antigua con la medicina".

7. La "segunda vocación" como estrategia de afrontamiento. El sentimiento de estar quedando obsoleto lleva a muchos médicos a renovarse, a explorar nuevos intereses y actividades dentro de la esfera profesional. El descubrimiento de una "segunda vocación" juega un papel relevante: "También tengo una segunda vocación. He tenido la posibilidad de hacer gestión, de hablar con los empresarios de igual a igual, del 'riesgo persona'. Generé efectos que creí no eran posibles". La preocupación por los más jóvenes se transforma en motivación renovada: "La docencia, formar nueva gente, es muy satisfactorio". "Buscar otras alternativas distintas a la medicina, la cual te toma la vida. Me dedico a la docencia en bioética, a formar comités de ética".

8. Estilo de vida y estrategia de afrontamiento. Desde luego, en el bienestar del médico juega un rol principal la calidad de la relación médicopaciente ${ }^{5}$. Pero tampoco hay que dejar de lado el rol del ejercicio físico, de la buena nutrición, de la práctica del sexo, del contacto con la naturaleza, de los viajes y hobbies, los deportes, el manejo del tiempo libre, la participación en redes sociales y, no por último menos importante, del cultivo de la amistad. Frente a estos desafíos, muchos médicos buscan maneras de mantener en alto los ideales que los llevaron a la medicina, conservando un balance con otros intereses y valores. Por cierto, esta búsqueda del equilibrio es un desafío que dura toda la vida, desde la escuela de medicina hasta la jubilación ${ }^{19}$. El que las estrategias varían en diversos países y culturas, lo demuestra un estudio llevado a cabo por un periodista inglés y un médico australiano ${ }^{20}$, que compara las estrategias que médicos británicos y australianos despliegan para mantener el bienestar subjetivo. Por su parte, nuestros médicos entrevistados tienden a "balancear la vida profesional y la vida personal... "a planear el tiempo y los presupuestos... y a tener una visión clara del sentido de la vida", o a "considerar menos ambiciosamente la medicina: Mi actividad como médico es sólo una más de las posibilidades de servicio al ser humano. Tampoco espero mucha retribución económica o social". Actividades extra profesionales también juegan un rol: "Uno tiene que buscar ayuda en otra parte... también en el jazz". "Estoy metido en un proyecto, en otro estudio; también leer otras cosas, hacer deporte". Y, por supuesto, también es importante el tiempo de descanso y ocio: "Planifico las vacaciones: salir a comer con la mujer y los hijos regularmente, irse fuera de Santiago por lo menos una vez al mes, salir de vacaciones dos veces al año".

9. Factores organizacionales internos y bienestar. Mientras los factores externos que causan estrés en las organizaciones de servicios de salud son 
bien conocidos, se reconocen poco los poderosos efectos adversos de factores internos, tales como el estilo de liderazgo y gestión y las políticas administrativas $^{21}$. Las organizaciones influencian al individuo que trabaja en ellas modelando sus percepciones, pensamientos, sentimientos, expectativas y conductas. Las organizaciones de salud pueden provocar alienación, pasividad y miedo en sus miembros, al dirigir la atención hacia la información objetiva, el control y la detección de problemas, alejándola de la información subjetiva, las relaciones humanas, y la valoración de logros y fortalezas. Cualquier miembro de una organización, particularmente sus líderes, puede promover una cultura organizacional saludable a través de la atención cuidadosa en la comunicación, las relaciones interpersonales, la toma de conciencia y el significado simbólico de políticas y conductas. En nuestro estudio, fueron los médicos que trabajan en servicios de urgencia y en atención primaria los más conscientes de la importancia del clima organizacional interno: "Acá es un grupo de apoyo, este grupo humano es un refugio, humor..., es tu segunda familia". "El trabajo en equipo, el apoyarse mutuamente entre colegas, tanto a nivel profesional como emocional". "El formar grupos de trabajo me ha ayudado a enfrentar los cambios, a no sentirme 'sólo contra el sistema'".

10. Identidad gremial, acciones políticas y bienestar. Desde luego, muchas de las fuentes de estrés y de burnout en medicina pueden retrotraerse al clima cultural y político más amplio en el que los médicos trabajan, y ser un reflejo de éste. Los médicos tienen, eso sí, la oportunidad de llegar a ser agentes sanadores en la sociedad, tomando primero conciencia de las fuentes del propio estrés para tomar entonces una posición de liderazgo que promueva valores y desarrolle métodos de estructurar la vida y el trabajo, de

\section{REFERENCIAS}

1. FirTh-Cozens J. Doctors, their well-being, and their stress. BMJ 2003; 326: 670-1.

2. JimÉNEZ JP. Bienestar y satisfacción de los médicos con el ejercicio de su profesión en un sistema de salud en proceso de reforma. Rev Méd Chile 2004; 132: 635-6. manera que permitan el florecimiento del espíritu. Por otro lado, aun cuando el bienestar médico puede comenzar con la puesta en práctica de valores y opciones individuales, su sostenimiento también requiere de acción y organización políticas. Éstas son necesarias para confrontar estructuras no saludables que conducen a los servicios de atención de salud a un encantamiento con las ganancias y el lucro, a expensas del servicio a los usuarios y de la moral de los proveedores de salud. La acción organizada tiene el triple potencial de ser más efectiva en cambiar sistemas, en aumentar la sensación de control y dominio sobre fuerzas complejas y en crear cohesión y apoyo grupal, todos componentes importantes del bienestar ${ }^{22}$. Los médicos jóvenes que trabajan en atención primaria parecen estar más conscientes de esto, al buscar activamente el "fortalecimiento de la actividad gremial como método de defensa frente a un medio más agresivo"; y la formación de asociaciones: "Formación de alianzas con entidades aseguradoras para mantener adecuadas plazas de pacientes y niveles de renta".

\section{CONCLUSIONES}

Nuestro estudio preliminar encontró una diversidad de estrategias que los médicos aplican para enfrentar la crisis en la profesión. Los médicos mayores mostraron el mayor grado de resignación pasiva y de conductas de escape. En cambio, los jóvenes que trabajan en atención primaria enfrentan la situación de una manera activa, intentando agruparse, estudiando y ejerciendo acciones en el medio. En todos los grupos se valora la necesidad de reflexionar sobre el significado y dirección de los cambios, lo que habla de una falta de oportunidades de diálogo y reflexión entre los médicos.

3. JimÉnEz J. Cambios en el entorno del trabajo médico. Rev Méd Chile 2004; 132: 637-42.

4. Horwitz N. El cambio de la práctica médica. Desafíos psicosociales para la profesión. Rev Méd Chile 2004; 132: 768-72.

5. BAsCuÑán ML Cambios en la relación médicopaciente y nivel de satisfacción de los médicos. Rev Méd Chile 2005; 133: 11-6. 
6. Schlozman S, Groves JE, Weisman AD. Coping with illness and psychotherapy of the medically ill. En: Stein ThA, Fricchione GL, Cassem NH, Jellinek MS, Rosenbaum JF, eds. Massachusetts General Hospital. Handbook of General Hospital Psychiatry. Philadelphia: Mosby 2004; 61-8.

7. KASSIRER J. Editorial. NEngl J Med 1998; 339: 1543-5.

8. Christensen JF, Feldman Mitchell D. A call for papers on physician well-being. West J Med 2000; 172: 293.

9. MyeRs MF. The well-being of physician relationships. West J Med 2001; 174: 30-3.

10. GABBARD G. The role of compulsiveness in the normal physician. JAMA 1985; 254: 2926-9.

11. MiLeR FE. Challenging and changing stress producing thinking. West J Med 2001; 174: 49-50.

12. EPSTEIN RM. Just being. West J Med 2001; 174: 63-5.

13. RaBow MW, McPheE SJ. Doctoring to Heal: Fostering well-being among physicians through personal reflection. West J Med 2001; 174: 66-9.
14. CLEVER LH. A checklist for making good choices in trying -or tranquil- times. West J Med 2001; 174: 41-3.

15. Charles SC. Coping with a medical malpractice suit. West J Med 2001; 174: 55-8.

16. McPhee SJ. Letter from the Abbey. West J Med 2001; 174: 73-5.

17 CU山FoRd L. Psycho spirituality will benefit healthcare staff. BMJ 2002; 324: 1523-4.

18. Myers DG. The funds, friends, and faith of happy people. Am Psychol 2000; 55: 56-67.

19. Suchman AL, Ramamurthy G. The well-being of clinicians. En: Feldman MD, Christensen JF, eds. Behavioral Medicine in Primary Care: a Practical Guide. New York: Appleton \& Lange; 1997.

20. Cross P, Brownscombe J. The British and Australian view of well-being. BMJ 2003; 326: 112.

21. Suchman AL. The influence of health care organizations on well-being. West J Med 2001; 174: 43-7.

22 Editor's pick. West J Med 2001; 174: 1. 